\title{
Gender Difference in Factors Affecting Quality of Sleep among Community Dwelling Elders
}

\author{
Lee KyuEun, Kim NamSun and Han SongHee \\ Department of Nursing, Catholic Kwandong University \\ Beomil-ro 579beon-gil, Gangneung-si, Gangwon-do, Korea \\ nskim@cku.ac.kr
}

\begin{abstract}
This study was done to identify the relationship of perceived health status, stress, pain and quality of sleep and to identify predictors of sleep quality for elderly people for each gender. Prevalence of sleep disturbance was $74.8 \%$ (male elders and $81.6 \%$ (female elders). Female elders were significantly lower perceived health status score $(t=2.19, p=.029)$, higher stress score $(t=2.93, p=.004)$, higher pain score $(t=-4.04, p<.001)$, than male elders. In multiple regression analysis, perceived health status, stress, spouse with or without were significant predictors and accounted for $17.6 \%$ of male elders' sleep quality. For female elders, stress was significant predictor and accounted for $12.0 \%$. Findings of this study provide a comprehensive understanding of sleep quality and related factors for elders in Korea. Also, these results could be used in developing sleep promotion program.
\end{abstract}

Keywords: Aged, Sleep, Pain, stress, Health

\section{Introduction}

Elders is the age group that reports sleep disturbance; $70 \%$ of elders suffer from sleep disturbance (Lindstrom, Andersson, Lintrup, Holst, \& Berglund, 2012). Aging accompanies changes in circadian rhythm and thus changes in sleep pattern, causing sleep problems such as difficulty falling asleep, frequent awakening, difficulty staying asleep, early awakening, lowering of sleep satisfaction, difficulty staying awake during the day, memory loss, and difficulty concentrating (American Academy of Family Physician, 2005).

Elders with sleep disorders run into sick leaves, emergencies, hospitalization, and other medical services (Novak, Musci, Shapiro, Rethelyi, \& Kopp, 2004). Moreover, elders' inappropriate sleep significantly increased morbidity rate and mortality rate, and compared to those without sleep disturbance, those suffering from sleep disturbance had lower quality of life and showed depression and anxiety more often (Neikrug \& Ancoil-Israel, 2010). Therefore, there is a need for understanding of changes in elders' sleep pattern accompanied by aging and a solution to improve elders' sleep quality is called for.

Elders do not belong to a homogeneous group, but a heterogeneous group that exhibits different characteristics, based on criteria such as gender, social class, and age, which are presumed to affect the sleep quality. Therefore, there is a need to offer helps to improve elders' sleep quality via analyzing the degree of sleep quality and the factors affecting sleep quality by gender, social class, and age.

The researches regarding elders' sleep up to date include researches on sleep pattern, on sleep quality, and on sleep disturbance; there are also accumulated research results on elders' health status, stress and pain. Thus, this research aims to analyze the difference between male 
and female elders in all factors that presumably affect sleep quality, including general variables such as perceived health status, stress, pain, and demographic characteristics.

\section{Methods}

\subsection{Setting and Samples}

Samples were randomly selected from elders welfare centers and senior citizen centers in City A and City G, Korea. The participants comprised 111 male elders and 190 female elders. Data were gathered from November 5th, 2013 and January 27th, 2014.

\subsection{Measures}

The perceived health status scale (Speak, Cowart, and Pellet, 1989) was used to measure health status. The stress scale (Lee, 1999) was used to measure the level of stress of elderly. Pain level was measured using Modified Visual Analogue pain rating Scale (MVAS) from (no pain) to 10(maximum pain). The Korean version of the Pittsburgh Sleep quality Index (Sohn, Kim, Lee, and Cho, 1989) was used to measure quality of sleep.

\subsection{Data Analysis}

Data were analyzed by using percentage, mean, standard deviation, Chi-square test, t-test, Pearson's Correlation Coefficient, stepwise multiple regression with the SPSS WIN 20.0 program.

\subsection{Ethical consideration}

Approval for the study was obtained from the Catholic Kwandong University Institutional Review Board.

\section{Research Results and Discussions}

The main title (on the first page) should begin $13 / 16$ inches (7 picas) from the top edge of the page, centered, and in Times New Roman 14-point, boldface type. Capitalize the first letter of nouns, pronouns, verbs, adjectives, and adverbs; do not capitalize articles, coordinate conjunctions, or prepositions (unless the title begins with such a word). Please initially capitalize only the first word in other titles, including section titles and first, second, and third-order headings (for example, "Titles and headings" — as in these guidelines). Leave two blank lines after the title.

\subsection{The Gender Difference in Prevalence of Sleep problems of Elders}

In Pittsburgh Sleep Quality Index, the sleep disturbance incident over the cutoff of 5 points among males and females were $74.8 \%$ and $81.6 \%$ respectively, and there was no significant difference between males and females. The prevalence of sleep problem among subjects was $74.8 \%$ (male elders) and $81.6 \%$ (male elders).

\subsection{The Gender Difference in Sleep Quality of Elders}

The average of male elders' sleep quality was $6.97 \pm 3.11$ point and the average of female counterpart was $7.44 \pm 3.18$ point; male elders had tendency to take less time to fall asleep, to 
have higher sleep efficiency, and to have higher sleep quality due to less interruptions during sleep.

However, Subjective sleep quality, sleep duration, use of sleeping medication, and daytime dysfunction were no difference between male and female elders.

\subsection{The Gender Difference in Correlation between Sleep Quality and Other Variables} of Elders

The sleep quality is negatively correlated to perceived health status in both male $(\mathrm{r}=-.28$, $\mathrm{p}=.003$. ) and female elders $(\mathrm{r}=-.27, \mathrm{p}=.003)$, and thus, the worse one's perceived health status was, the worse his or her sleep quality was. This result conforms to the report by Neikrug and Ancoil-Isreal (2010) that elders' inappropriate sleep is related to disease morbidity rate and death rate.

For both male elders $(\mathrm{r}=.36, \mathrm{p}<.001)$ and female elders $(\mathrm{r}=.30, \mathrm{p}<.001)$, stress has shown a positive correlation with sleep quality: the more stress, the worse sleep quality. Aanes and others (2011) reported that stress caused by inappropriate social relation or loneliness and sleep problems have a strong correlation and supported this research result.

Moreover, female elders' sleep quality is positively correlated to pain $(r=.24, p=.001)$ : the worse her perceived health status is, the worse her pain is, the worse her sleep quality is. That is, pain was related to female elders' sleep quality but not to male elders' sleep quality. The sleep disorders were more often reported by the subjects who have experienced pain in four weeks than the subjects who have not. Considering the report that there was a correlation between pain and sleep disorders (Lindstrom et al., 2012), it seems that female elders with higher pain score exhibit a higher correlation between pain and sleep disorders than male elders with lower pain score. Taking into account the fact that most elders suffer from several chronic conditions, efficient nursing intervention for pain management will help improve elders' sleep quality.

\subsection{The Gender Difference in Factors Affecting Quality of Sleep of Elders}

For male elders, stress was the most powerful factor, and three factors combined with perceived health status and the presence of spouse explained $17.6 \%$ of sleep quality. The presence of spouse is the factor that demonstrated difference in quality of life more among males than among females. According to Jeon's research (2008), males without spouse had approximately 3 to 5 times lower quality of life than those with. This result is interpreted to be due to traditional Korean society, in which the gender roles are clearly distinguished (Jeon, 2008).

For female elders, stress alone explained $12 \%$ of sleep quality. However, stress variable had a greater sleep quality among male elders than among female elders. This is due to that female elders are better supported by family members or friends than male elders are. In case of social support, male elders mainly depend on spouses, but female elders tend to rely on same-sex friends (Knodel \& Ofstedal, 2003), and there was a gender difference in stressreducing strategy.

\section{Conclusion}

Since stress-reducing strategy is necessary for sleep quality improvement of both male and female elders, a program to reduce stress in the whole local community needs to be developed and applied. 
However, the fact that stress besides, sleep quality of male elders and female elders are affected by different factors, implies that different strategies to improve elders' health are needed for different genders.

Since social relation network especially affects the subjective health status significantly, it is expected of male elders without spouses to expand social relation network, and thus, reduce the negative effect caused by perceived health status and without spouse in order to improve sleep quality.

\section{References}

[1] M. M. Aanes, J. Hetland, S. Pallesen and M. B. Mittelmark, "Does loneliness mediathe stress-sleep quality relation?", The Hordaland health study. International Psychogeriatric, vol. 23, no. 6, (2011), pp. 994-1002.

[2] American Academy of Family Physician, Sleep changes in older adults. American Family Physician, vol. 72, no. 7, (2005), pp. 13-15.

[3] G. S. Jeon, "Gender difference in social factors of health in later life", Journal of Korean Gerontological Society, vol. 28, no. 3, (2008), pp. 459-475.

[4] J. Knodel and M. B. Ofstedal, "Gender and aging in the developing world: where are the men?", Population and Development Review, vol. 29, no. 4, (2003), pp. 677-698.

[5] H. S. Lee, "Factors of sleep disturbance and sleep patterns according to age in older hospitalized women patients", Journal of Korean Academy of Fundamentals of Nursing, vol. 18, no. 2, (2011), pp. 186-194.

[6] V. Lindstrom, K. Andersson, M. Lintrup, G. Holst and J. Berglund, "Prevalence of sleep problems and pain among the elderly in Sweden", The Journal of Nutrition Health \& Aging, vol. 16, no. 2, (2012), pp. 180-183.

[7] A. B. Neikrug and S. Ancoil-Israel, "Sleep disorders in the older adults -A mini-review", Gerontology, vol. 56, (2010), pp. 181-189.

[8] M. Novak, I. Mucsi, C. M. Shapiro, J. Rethelyi and M. S. Kopp, "Increased utilization of health services by insomnias: An epidemiological perspective", Journal of Psychosomatic Research, vol. 56, (2004), pp. 527536.

[9] S. Park, S. Cho and S. Jang, "Positive and negative influence of social network on self-rated health and its gendered pattern", Korean Journal of Health Education and promotion, vol. 28, no. 4, (2011), pp. 39-49.

[10] S. H. Sohn, D. H. Kim, M. Y. Lee and Y. W. Cho, "The reliability and validity of the Korean version of the Pittsburgh sleep quality index”, Sleep Breath, vol. 16, (2012), pp. 803-812.

[11] D. L. Speake, M. E. Cowart and K. Pellet, "Health perceptions and lifestyles of the elderly", Research in Nursing \& Health, vol. 12, (1989), pp. 93-100.

* Corresponding author: Kim, NamSun, Ph.D.

Department of Nursing, Catholic Kwandong University

Beomil-ro 579beon-gil, Gangneung-si, Gangwon-do, Korea

E-mail: nskim@cku.ac.kr

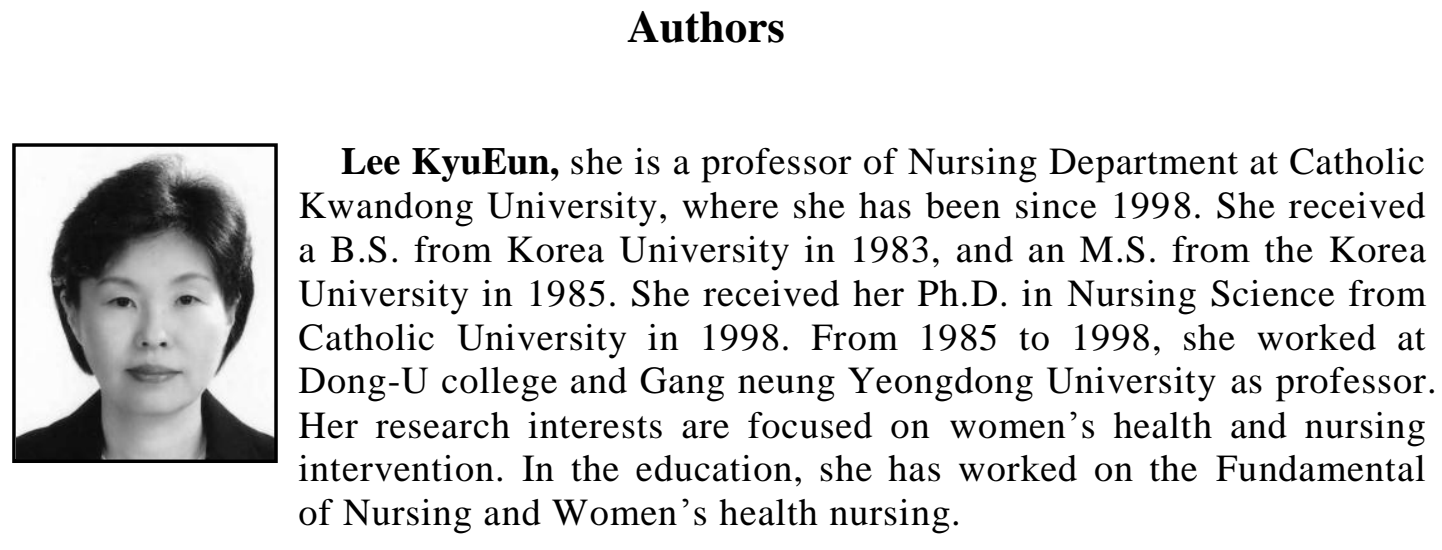




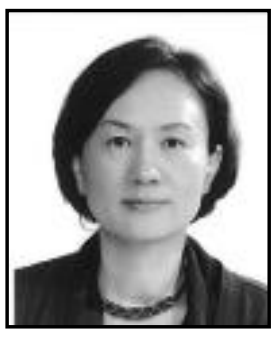

Kim NamSun, she is a professor of Nursing Department at Catholic Kwandong University, where she has been since 1995. She received a B.S. from Ewha Womens University in 1985, and an M.S. from the Ewha Womens University in 1987. She received her DNsc. from Ewha Womens University in 1994. From 1991 to 1994, she worked at Young Dong College as professor. Her research interests are focused on the effects of nursing intervention in middle-aged Women.

And also, she is interested the improvement of student's personal growth initiative with enneagram.

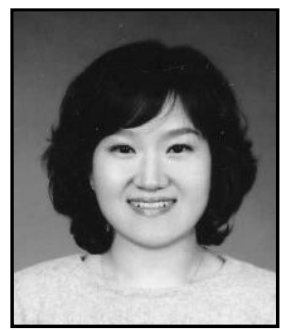

Han SongHee, she is a PhD student of Nursing Department at the Chung-Ang University. She received a B.S. from Catholic Kwandong University in 1999, and an M.S. from the Catholic Kwandong University in 2004. Currently, she gives lectures in some universities located in Gangwon-do. Her research interests are focused on older adults's health and health promotion behavior in community. 
International Journal of Bio-Science and Bio-Technology Vol.7, No.1 (2015) 